\title{
Turning down insulin signaling
}

\author{
Morris J. Birnbaum
}

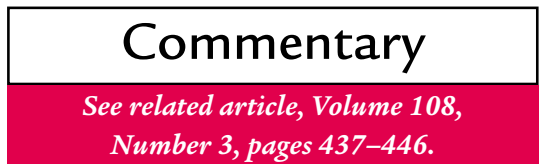

Howard Hughes Medical Institute and The Cox Institute at the University of Pennsylvania School of Medicine, Philadelphia, Pennsylvania, USA

Address correspondence to: Morris J. Birnbaum, Howard Hughes Medical Institute, Building CRB, Room 322 , 415 Curie Boulevard, Philadelphia, Pennsylvania 19104, USA. Phone: (215) 898-5001; Fax: (215) 573-9138; E-mail: birnbaum@mail.med.upenn.edu.

J. Clin. Invest. 108:655-659. DOI:10.1172/JCI200113714.

In recent years, much has been written about the importance of diabetes mellitus, both as a cause of widespread morbidity and mortality and in terms of the resultant overwhelming health care costs. In an analysis of worldwide diabetes in 1994, the World Health Organization reported that the agestandardized prevalence in European populations varied from $3 \%$ to $10 \%$, while more restricted populations demonstrated prevalence of up to $50 \%$ (1). Over the same period, approximately 10.2 million people with diagnosed diabetes mellitus resided in the US, but another 5.4 million individuals with diabetes went undiagnosed (2). Perhaps even more impressive, these numbers have been estimated to represent as much as a $50 \%$ increase over the prevalence in equivalent populations during the previous decade. In 1997, health care expenditures attributable to diabetes in the US were estimated to be $\$ 98$ billion (3). Undoubtedly, this is due not only to the widespread prevalence of the disease, but also to its chronic nature and disabling complications, affecting cardiovascular, renal, visual, and neurological function. Thus, perhaps the lack of an even more intense research effort into the pathophysiology of diabetes can only be attributed to the difficulty inherent in making progress in the study of this complex, multisystem disease. For these reasons, it is particularly important that in two recent articles - one appearing in the current issue of the journal Science and the other a recent issue of the JCI - Steven Shoelson, Gerald Shulman, and their colleagues present a new hypothesis, which not only purports to explain the insulin resistance of type 2 diabetes mellitus but also offers a clear basis for the development of novel therapeutics $(4,5)$.

Over $90 \%$ of diabetes mellitus is accounted for by what is now called the type 2 variant. Unlike type 1 dia- betes, for which there is a reasonable consensus that the disease results from autoimmune destruction of insulin-secreting pancreatic $\beta$ cells, the etiology of type 2 diabetes remains a bit uncertain. Most investigators and clinicians agree that genetic and environmental factors contribute and that obesity is a frequent if not essential antecedent of the disease. Perhaps the most heated debate among diabetes researchers has concerned the nature of the primary inciting metabolic event, that is, whether it represents a disturbance in the normal pattern of insulin secretion or abnormalities in the action of insulin in peripheral tissues (6). Experiments in which defects in insulin secretion or action have been selectively introduced into mice by the modification of single or multiple genes have been surprisingly unhelpful at resolving this issue. Perhaps these genetic studies only serve to emphasize the multi-organ system nature of diabetes mellitus, in which several defects are required to elicit sufficient dysfunction to overwhelm physiological compensatory mechanisms and produce diabetes.

Nevertheless, investigators have postulated a reasonable series of events to explain the evolution of type 2 diabetes (7). According to this model, peripheral insulin resistance represents the earliest event, but this is initially compensated by enhanced insulin secretion. Later, the $\beta$ cell no longer keeps pace with the increased needs, and a relative lack of insulin is followed by an absolute deficiency of the hormone. At about the same time, the liver develops insulin resistance, thus leading to accelerated production of glucose. Whatever the precise sequence of the events by which impaired glucose tolerance matures to diabetes, there is little doubt that insulin resistance represents an important component of the fulminant disease.

\section{Insulin signaling then and now}

To appreciate studies of the pathophysiology of insulin resistance, one must be familiar with the state of knowledge of insulin signal transduction and how these pathways link to biological outputs. Twenty years ago, when it became clear that the insulin receptor not only conferred specificity in terms of hormone binding, but also possessed intrinsic protein tyrosine kinase activity, the predominant hypothesis was that insulin signaled by initiating a cascade of enzyme reactions. This concept was largely influenced by the biochemistry of glycogen metabolism, in which one kinase phosphorylates another, producing a linear path of amplifying reactions. Thus, it was expected that the insulin receptor would act by transferring a phosphate group to a tyrosine residue on another enzyme, thereby influencing the latter's activity.

A radical change in thinking was inspired by the discovery that the major sites phosphorylated by the PDGF receptor were not on a downstream signaling molecule but were instead specific tyrosine residues in the cytoplasmic domain of the PDGF receptor itself. Moreover, these covalent modifications did not alter the catalytic activity of the receptor but rather provided docking sites for the recruitment of a number of proteins, each capable of initiating a distinct signaling pathway. Thus, by the time a cDNA encoding the first major substrate of the insulin receptor, insulin receptor substrate 1 (IRS-1), was cloned and the primary structure of the protein deduced, it came as no surprise that it acted as a scaffolding protein (8). The predicted sequence contains numerous tyrosine residues in contexts that made them likely candidates for phosphorylation by the insulin receptor. Moreover, these sites, when phosphorylated, recruit several 
key signaling molecules by means of binding to the latter's Src homology 2 (SH2) domains.

This scheme immediately points out one of the distinguishing characteristics of signaling by insulin and related peptides. Unlike PDGF, for example, insulin promotes the assembly of a stable signaling complex not on the receptor itself, but on a separate scaffold, that is, IRS-1, or one of its related proteins. The explanation most frequently invoked to explain such specialization is the need to target insulinregulated signaling molecules to a critical subcellular locale, for which IRS- 1 serves as an escort. In reality, the proof of such a model is wanting, even though the IRS proteins do appear to move among subcellular regions after phosphorylation. The sites of phosphorylation emerged as another potential control point for regulating this pathway. Whereas purified insulin receptor phosphorylates IRS-1 exclusively on tyrosine residues, treatment of the intact cell with hormone leads to extensive phosphorylation on serine and threonine. This finding suggests that activation of the insulin receptor stimulates downstream serine/threonine kinases that act on IRS-1, perhaps as part of a negative feedback loop that suppresses insulin signaling.

\section{Defining the relevant aspects of insulin signaling}

Because insulin stimulation leads to numerous changes in cellular metabolism and growth, the signaling events that relate to the glucose transport and the other physiological actions of insulin have been difficult to identify. The problem of how to link particular phosphorylated species or particular IRS-1-associated proteins to these events has become increasingly acute as techniques have improved to define candidate molecules. In spite of the lack of clarity regarding the normal pathways mediating insulin-stimulated glucose uptake into muscle and fat, a considerable effort has been invested in pinpointing the site of abnormality in insulin-resistant states - a daunting task, in part because of the interplay of genetic and environmental factors, upon which acquired physiological changes are superimposed as the disease progresses. Thus, one could imagine that some critical but subtle event impairs insulin signaling in muscle, leading to impaired glucose uptake following a meal. In an otherwise normal individual, this produces an appropriate reactive hyperinsulinemia, which downregulates peripheral insulin receptors and initiates an assortment of poorly understood negative feedback pathways. At this point, it becomes virtually impossible to distinguish between the initial insult and the compensatory changes.

Nevertheless, work from many sources supports the notion that the lipid kinase phosphatidylinositol $3^{\prime}$-kinase (PI 3'-kinase) represents an obligate intermediate in the pathway by which insulin regulates diverse metabolic functions, including glucose transport in muscle and adipose tissue. This conclusion is based on studies of the effects of PI $3^{\prime}$-kinase inhibitory drugs, inhibitory mutants of PI 3'-kinase, or neutralizing antibodies introduced into cells or tissues. What lies downstream of PI 3 '-kinase is considerably less certain, although several serine/threonine kinases, such as Akt (also known as protein kinase B) and the atypical protein kinase $C$ enzymes, have been suggested $(9,10)$. In addition, another pathway, whose operation is independent of both the IRS proteins and PI 3'-kinase, has recently been postulated as critical to insulin action in adipocytes (11). The terminal event in the regulation of glucose transport is much better established: A specialized hexose carrier, Glut4, is sequestered within the interior of the cell, to be redistributed to the surface in cells exposed to insulin; this hormone-dependent Glut4 translocation results in an increase in transporter number in the plasma membrane, which appears sufficient to explain most or all of the augmentation in glucose flux evoked by insulin (ref. 12; see also the article by Tong et al., this issue, ref. 13).

One mechanism that has attracted a fair amount of attention over the years as a potential site of regulation of insulin signaling is IRS-1 serine phosphorylation. Exposure of cells to insulin for prolonged periods of time results in increased phosphorylation of IRS-1 on serine and threonine residues, a modification that is associated with a dramatic drop in both insulin-stimulated tyrosine phosphorylation of IRS-1 and its association with PI 3'-kinase. Multiple kinases - mitogen-activated protein kinase (MAPK/ERK), c-Jun $\mathrm{NH}_{3}$-terminal kinase (JNK), a mammalian target of rapamycin-dependent kinase, atypical protein kinase C, PI 3'-kinase, and others - are known that can phosphorylate IRS- 1 and IRS- 2 on serine or threonine residues in the intact cell, but the importance of these enzymes in normal or diabetic tissue has been difficult to establish (14-19). Similarly, several researchers have invested a fair effort into identifying the critical residues whose phosphorylation is responsible for decreasing the effectiveness of IRS-1 as an insulin receptor substrate. No clear picture has yet emerged of the pathway that governs IRS serine/threonine phosphorylation.

Two lines of investigation regarding putative pathways mediating IRS phosphorylation are particularly intriguing, since in both cases the proximal stimuli have been implicated independently as critical to the development of insulin resistance. The first pathway involves tumor necrosis factor TNF- $\alpha$, which was identified about 15 years ago as an agent that induces hemorrhagic necrosis in some transplantable tumors and, independently, as a factor responsible for the development of cachexia. More recently, TNF- $\alpha$ has been shown to be increased in adipose tissue from obese, insulin-resistant humans and animals. Neutralization of TNF- $\alpha$ reverses the insulin resistance in animals, a finding that has yet to be replicated in diabetic humans $(20,21)$. TNF- $\alpha$ apparently abrogates much of insulin signaling by promoting increased serine/threonine phosphorylation of IRS-1 (22), which not only reduces the ability of IRS-1 to act as a substrate for the protein kinase activity of the insulin receptor, but actually converts IRS-1 into an inhibitor of the receptor kinase (Figure 1a). However, the remarkable diversity of signaling pathways regulated by TNF- $\alpha$ has made it particularly difficult to pinpoint which activity is responsible for phosphorylating IRS- 1 and inhibiting subsequent activation by insulin.

The second model for the development of insulin resistance that has garnered much recent interest relates to the involvement of lipids in modulating glucose metabolism. One characteristic metabolic abnormality in peo- 
a

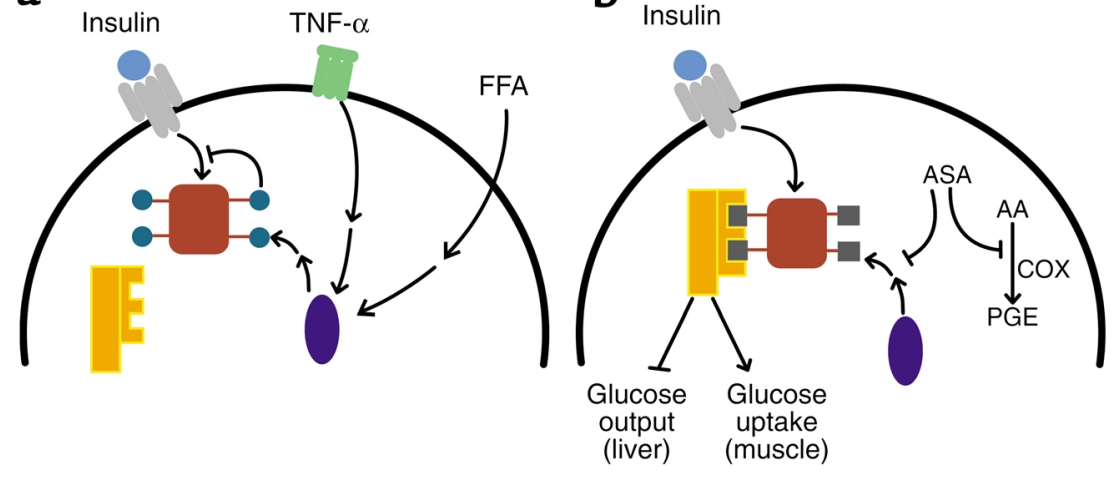

b

\section{Figure 1}

Effect of salicylates on insulin signaling during insulin resistance. (a) Normally, the occupied insulin receptor phosphorylates scaffold proteins, such as the IRS-1, on critical tyrosine residues. However, in insulin-resistant states, a number of agents, such as the cytokine TNF- $\alpha$ or circulating FFAs, lead through intermediary signaling pathways to the activation of IKK, which in turn indirectly increases the number of phosphorylated serine and threonine residues (indicated by blue circles) on IRS-1. This modification blocks the tyrosine phosphorylation and converts IRS-1 into an insulin receptor inhibitory protein. (b) In the presence of salicylates, IKK activity is inhibited, reducing the IRS-1 serine/threonine phosphorylation and allowing IRS-1 to be phosphorylated on tyrosine. These phosphorylated tyrosine residues (black squares) serve as binding sites for a number of signaling molecules, most importantly PI 3'-kinase, which initiate signaling pathways regulating metabolism. Aspirin (ASA) also inhibits cyclooxygensases (COX) to prevent the generation of inflammatory prostaglandins (PGE) from arachidonic acid (AA) in a pathway unrelated to the effects of the drug on insulin action.

ple with either impaired glucose tolerance or type 2 diabetes mellitus is an elevation in the level of circulating free fatty acids (23). Although the earliest attempts to establish fatty acids as a cause of insulin resistance were unsuccessful, it is now clear that prolonged infusions of fatty acids effectively inhibit muscle glucose transport and oxidation (24). The so-called Randle hypothesis (25) proposed that fat metabolism suppressed glucose oxidation by inhibition of glycolysis, but recent studies have shown that a more important site of action is at the level of glucose transport. This points to some still-obscure regulatory circuit by which fat metabolism or content in muscle directly leads to suppression of insulin signaling or Glut4 translocation. Again, infusion of fatty acids also results in increased serine/threonine phosphorylation of IRS- 1 and a reduction in IRS-1-associated PI 3'-kinase in muscle (26) (Figure 1a).

In spite of the many examples in which serine/threonine phosphorylation of IRS- 1 begets defects in proximal insulin signaling, there still

remain several problems with invoking this phenomenon as a critical, clinically important cause of insulin resistance. First, as noted above, although serine/threonine phosphorylation of IRS-1 is associated with insulin resistance in vivo, it has generally not been possible to establish the altered state of IRS- 1 as the cause of a systemic metabolic abnormality. Second, there are several striking examples in which disruption of the IRS-1-associated PI $3^{\prime}$-kinase signaling complex results in remarkably little inhibition of downstream signaling pathways or biological responses $(27,28)$. Lastly, studies testing the coupling of IRS-1 serine/threonine phosphorylation to the inhibition of PI 3'-kinase virtually all fall short of demonstrating functional consequences at the level of hexose uptake or Glut4 translocation. Without such data, all models must be viewed with caution.

\section{Salicylates and insulin responsiveness}

Now, with two recent publications, Shoelson and his colleagues have pro- posed a novel, and particularly attractive, pathway by which diverse conditions might lead to serine/threonine phosphorylation of the IRS proteins and thus to the inhibition of insulin signaling $(4,5)$. Shoelson's ideas were inspired by the periodic, recurrent reports, extending far back in the literature, suggesting that treatment of diabetic patients with high doses of salicylates, including sodium salicylate and aspirin, produces a remarkable amelioration of glucose intolerance. As an initial step, Yuan et al. simply confirmed this observation, administering salicylates under controlled conditions to several rodent models of diabetes (4). The earlier human studies had not addressed the question of mechanism, but glucose tolerance tests in the mice and rats quickly pointed to the site of action. The reductions in both blood glucose and insulin levels after therapy were pathognomonic of an improvement in insulin action as opposed to enhanced secretion; this was confirmed by an insulin tolerance test, in which the hormone was administered and the resultant decrease in blood sugar assessed. Interestingly, this improvement in glucose tolerance was matched by equivalent changes in the animals' lipid profiles. In fact, reductions in circulating fatty acids actually preceded the alterations in blood glucose, at least raising the possibility that the latter were secondary to improvements in lipid metabolism.

The authors also confirmed that the well-recognized inhibition in insulindependent tyrosine phosphorylation of the insulin receptor and IRS-1 accompanying insulin-resistant states can be relieved by salicylates, apparently associated with a decrease in serine/threonine phosphorylation of IRS-1 in the liver (4). However, as noted above, it is impossible to establish cause and effect from such biochemical correlations. To approach this question, Shoelson and colleagues turned to several tissue culture models of insulin resistance. Here, they demonstrated clearly that salicylates relieve the insulin signaling defects caused by TNF- $\alpha$ or serine phosphatase inhibitors and that this correlates nicely with increases in both the electrophoretic mobility and the insulin-dependent tyrosine phosphorylation of IRS- 1 and IRS-2. 
Although the salicylate-induced recovery of these early signaling events in liver and adipose cell lines is striking, the authors have yet to link this to improvements in insulin's ability to regulate an authentic biological response, such as glycogen synthesis or glucose transport. In addition, while the tissue culture data add significant weight to the proposition that reductions in IRS serine/threonine phosphorylation precedes and thus may cause the defects in insulin action, one must keep in mind the limitations of in vitro models of organismal disease. In particular, these experiments do not establish whether the mechanism by which TNF- $\alpha$ produces insulin-signaling abnormalities in cultured cells relates to the development of true insulin resistance in obese or genetically predisposed animals. Nonetheless, the data presented by Kim et al. in a recent issue of the JCI lend considerable weight to the validity of the in vitro system (5). In this study, insulin resistance in striated muscle is generated by a lipid infusion that raises circulating FFA levels. Pretreatment with salicylates blocks the decrease in insulin responsiveness, and this response correlates with an increase in insulin-dependent IRS1 -associated PI $3^{\prime}$-kinase (5).

What then is the biochemical target of salicylates in these experiments? Here Shoelson, Shulman, and colleagues have zeroed in on the signaling pathway regulating NF- $\mathrm{KB}$. Under resting conditions, this transcription factor is held in an inactive state by binding to an inhibitory partner, IКB (29). Phosphorylation of the latter protein by IKB kinase (IKK) leads to the proteosome-catalyzed degradation of I $\kappa$ B, releasing NF- $\kappa$ B for translocation to the nucleus where it can influence the transcription of diverse genes. Two factors led to the model of salicylate function shown in Figure 1b. First, the NF- $\kappa \mathrm{B}$ signaling pathway is one of the major biochemical mediators of the actions of TNF- $\alpha$, which, as described above, is capable of inducing insulin resistance. Second, and perhaps more compelling, high concentrations of aspirin and salicylate directly inhibit the activity of IKK (30). In fact, Yuan et al. use nonsteroidal anti-inflammatory agent inhibitors of cyclooxygenases (COXs) to demon- strate that the effects of salicylates track most closely with their ability to inhibit IKK and not COX-1 or -2 (4). This is consistent with the high doses of aspirin required for effects in insulin sensitivity, as the antithrombotic effects of the drug are mediated by inhibition of COX at low doses (31). But most impressive are the results on mice heterozygous for a mutation engineered into the IKK $\beta$ gene, a lesion that interferes with expression of the protein. Suppression of IKK $\beta$ activity reverses much of the insulin resistance present in genetically obese mice, or that produced by high-fat feeding (4). In fact, disruption of a single IKK $\beta$ allele completely corrects both the skeletal insulin resistance caused by elevation in FFAs, and the biochemical defects in IRS- 1 tyrosine phosphorylation and activation of PI $3^{\prime}$-kinase. The remarkable correlation between the effects of salicylates and the genetic removal of IKK activity in several distinct models of insulin resistance provides the most compelling argument that activation of IKK leads to inhibition of insulin signaling by serine/threonine phosphorylation of IRS proteins and that this inhibition is critical to the development of insulin resistance.

\section{Challenges ahead}

A number of important questions need to be answered in the immediate future. Can the beneficial effects of aspirin on glucose tolerance in type 2 diabetics be reproduced under modern conditions, where it is possible to accurately assess the effects on insulin action in the periphery and liver, as well as insulin secretion? What is the precise biochemical pathway by which IKK leads to increased serine/threonine phosphorylation of IRS proteins? There are no data to indicate whether IKK interacts directly with IRS-1, nor even whether the response depends on the intervening activation of NF- $\mathrm{KB}$. Since IRS-1 is phosphorylated on so many sites in rather diverse sequence contexts, it is plausible that IKK affects the IRS phosphorylation state more broadly, perhaps by regulating removal of phosphate residues. Lastly, it still needs to be firmly established that the changes in IRS-associated PI 3 '-kinase represent the actual cause of impaired insulin responses, and not simply an accompanying biochemical event. However these details sort out, this new, provocative hypothesis will consume diabetes researchers' interest for some time to come and might well lead to important and novel therapeutic approaches to the treatment of type 2 diabetes mellitus.

1. King, H., and Rewers, M. 1993. Global estimates for prevalence of diabetes mellitus and impaired glucose tolerance in adults. WHO Ad Hoc Diabetes Reporting Group. Diabetes Care. 16:157-177.

2. Harris, M.I., et al. 1998. Prevalence of diabetes, impaired fasting glucose, and impaired glucose tolerance in US adults. The Third National Health and Nutrition Examination Survey, 19881994. Diabetes Care. 21:518-524.

3. 1998. Economic consequences of diabetes mellitus in the U.S. in 1997. American Diabetes Association. Diabetes Care. 21:296-309.

4. Yuan, M., et al. 2001. Reversal of obesity- and dietinduced insulin resistance with salicylates or targeted disruption of IKK $\beta$. Science. In press.

5. Kim, J.K., et al. 2001. Prevention of fat-induced insulin resistance by salicylate. J. Clin. Invest. 108:437-446.

6. Taylor, S.I., Accili, D., and Imai, Y. 1994. Insulin resistance or insulin deficiency. Which is the primary cause of NIDDM? Diabetes. 43:735-740.

7. DeFronzo, R.A. 1997. Pathogenesis of type 2 diabetes: metabolic and molecular implications for identifying diabetes genes. Diabetes Reviews. 5:177-269.

8. Sun, X.J., et al. 1991. Structure of the insulin receptor substrate IRS-1 defines a unique signa transduction protein. Nature. 352:73-77.

9. Summers, S.A., et al. 1999. Signaling pathways mediating insulin-stimulated glucose transport. Ann. NY Acad. Sci. 892:169-186.

10. Pessin, J.E., and Saltiel, A.R. 2000. Signaling pathways in insulin action: molecular targets of insulin resistance. J. Clin. Invest. 106:165-169.

11. Baumann, C.A., et al. 2000. CAP defines a second signalling pathway required for insulin-stimulated glucose transport. Nature. 407:202-207.

12. Birnbaum, M.J. 1992. The insulin-responsive glucose transporter. Int. Rev. Cytol. 137:239-297.

13. Tong, P., et al. 2001. Insulin-induced cortical actin remodeling promotes GLUT4 insertion at muscle cell membrane ruffles. J. Clin. Invest. 108:371-381.

14. Li, J., DeFea, K., and Roth, R.A. 1999. Modulation of insulin receptor substrate-1 tyrosine phosphorylation by an Akt/phosphatidylinositol 3-kinase pathway. J. Biol. Chem. 274:9351-9356.

15. Aguirre, V., Uchida, T., Yenush, L., Davis, R., and White, M.F. 2000. The c-Jun NH(2)-terminal kinase promotes insulin resistance during association with insulin receptor substrate- 1 and phosphorylation of Ser(307). J. Biol. Chem. 275:9047-9054.

16. Qiao, L.Y., Goldberg, J.L., Russell, J.C., and Sun, X.J. 1999. Identification of enhanced serine kinase activity in insulin resistance. J. Biol. Chem. 274:10625-10632.

17. De Fea, K., and Roth, R.A. 1997. Modulation of insulin receptor substrate-1 tyrosine phosphorylation and function by mitogen-activated protein kinase. J. Biol. Chem. 272:31400-31406.

18. Ravichandran, L.V., Esposito, D.L., Chen, J., and Quon, M.J. 2001. Protein kinase C-zeta phosphorylates insulin receptor substrate- 1 and impairs its ability to activate phosphatidylinositol 3kinase in response to insulin. J. Biol. Chem. 276:3543-3549.

19. Tanti, J.F., Gremeaux, T., Van Obberghen, E., and Le Marchand-Brustel, Y. 1994. Insulin receptor substrate 1 is phosphorylated by the serine kinase activity of phosphatidylinositol 3-kinase. Biochem J. 304:17-21. 
20. Hotamisligil, G.S., and Spiegelman, B.M. 1994. Tumor necrosis factor alpha: a key component of the obesity-diabetes link. Diabetes. 43:1271-1278.

21. Qi, C., and Pekala, P.H. 2000. Tumor necrosis factor-alpha-induced insulin resistance in adipocytes. Proc. Soc. Exp. Biol. Med. 223:128-135.

22. Hotamisligil, G.S., et al. 1996. IRS-1-mediated inhibition of insulin receptor tyrosine kinase activity in TNF-alpha- and obesity-induced insulin resistance. Science. 271:665-668.

23. Shulman, G.I. 2000. Cellular mechanisms of insulin resistance. J. Clin. Invest. 106:171-176.

24. Boden, G. 1997. Role of fatty acids in the pathogenesis of insulin resistance and NIDDM. Diabetes. 46:3-10.
25. Randle, P.J., Garland, P.B., Hales, L.N., and Newsholme, E.A. 1963. The glucose fatty acid cycle, its role in insulin sensitivity and the metabolic disturbances of diabetes mellitus. Lancet. 1:785-789.

26. Griffin, M.E., et al. 1999. Free fatty acid-induced insulin resistance is associated with activation of protein kinase $\mathrm{C}$ theta and alterations in the insulin signaling cascade. Diabetes. 48:1270-1274.

27. Staubs, P.A., Nelson, J.G., Reichart, D.R., and Olefsky, J.M. 1998. Platelet-derived growth factor inhibits insulin stimulation of insulin receptor substrate-1-associated phosphatidylinositol 3kinase in 3T3-L1 adipocytes without affecting glucose transport. J. Biol. Chem. 273:25139-25147. 28. Summers, S.A., et al. 1999. The role of glycogen synthase kinase 3beta in insulin-stimulated glucose metabolism. J. Biol. Chem. 274:17934-17940.

29. Karin, M., and Delhase, M. 2000. The I kappa B kinase (IKK) and NF-kappa B: key elements of proinflammatory signalling. Semin. Immunol. 12:85-98.

30. Yin, M.J., Yamamoto, Y., and Gaynor, R.B. 1998 The anti-inflammatory agents aspirin and salicy late inhibit the activity of I(kappa)B kinase-beta. Nature. 396:77-80.

31. Patrono, C., Patrignani, P., and Rodriguez, L.A 2001. Cyclooxygenase-selective inhibition of prostanoid formation: transducing biochemical selectivity into clinical read-outs. J. Clin. Invest. 108:7-13. 